\title{
Qualitative Examination of Daytime Monitoring and Selective Attention in Insomnia
}

\author{
Umair Akram ${ }^{1,2}$ [D $\cdot$ Shannon Kay ${ }^{1} \cdot$ Zoe Fonquerine $^{1}$
}

Received: 21 September 2018 / Accepted: 14 November 2018 / Published online: 22 November 2018

(c) The Author(s) 2018

\begin{abstract}
Purpose Insomnia is partly maintained by selective attention and monitoring for cues that indicate the presence of a poor night's sleep. However, little published work examining the role of selective attention in insomnia from a qualitative perspective exists.

Methods Eleven participants with DSM-5 insomnia disorder completed semi-structured interviews.

Results "Thematic analysis" revealed two prominent, yet interrelated, themes: impairment to daytime functioning and selective attention and monitoring. Perceived difficulty initiating and maintaining asleep during the biological night was accompanied by increased efforts during the day to complete mundane tasks. In addition, reports of consciously selectively attending to sleep-related cues on awakening were frequent, and for some this behaviour extends throughout the day. Conclusion The current outcomes provide first-person support for cognitive models of the disorder. Treatment approaches may wish to target and alleviate selective attention in insomnia.
\end{abstract}

Keywords Insomnia $\cdot$ Daytime impairment $\cdot$ Attentional bias $\cdot$ Interpretative bias $\cdot$ Tiredness $\cdot$ Qualitative

\section{Introduction}

Insomnia is a prevalent sleep disorder affecting up to $10 \%$ of adults at disorder level [1, 2]. It is characterised by difficulty with sleep initiation, maintenance and/or early morning awakening, and accompanied by significant impairment to daytime functioning. As a result, insomnia often leads to impaired quality of life [3]. Several theoretical cognitive models have explained proposed mechanisms influencing the development and maintenance of the disorder [4-6]. Here, insomnia is partly sustained by an attentional bias for negative sleep-related cues which may be internal (i.e., bodily sensations) or external (i.e., physical cues) [5, 6]. Sleep-specific anxiety then follows, which is said to be maintained by negative thoughts and beliefs concerning sleep, and worry

Umair Akram

u.akram@shu.ac.uk

1 Department of Psychology, Sociology and Politics, Sheffield Hallam University, Collegiate Crescent, Sheffield, SY S10 2BP, UK

2 Sleep and Circadian Neuroscience Institute, Nuffield Department of Clinical Neurosciences, University of Oxford, Oxford, UK concerning the daytime consequences of poor sleep. As a result, attentional resources are favourably allocated to the processing of sleep-related cues. On detection, a disorderconsistent interpretation follows, serving to further increase negative thoughts and beliefs concerning sleep, and worry concerning the daytime consequences of poor sleep; ultimately, a vicious thought cycle that is partly maintained by the sleep-related attentional bias.

A number of studies have examined the presence of sleeprelated attentional biases in insomnia using experimental tasks including the dot-probe, flicker, Posner, and the emotional Stroop tasks (see [19] for a review) [7-18]. Many of these studies suggest that poor sleepers and individuals with insomnia present an attentional bias for sleep-related stimuli [7-11, 13-15, 17]. Despite this, the evidence base advocating the presence of such a bias in insomnia remains mixed, [12, 16], [17] perhaps due to methodological differences regarding the specific task or population.

The aforementioned studies have been instrumental in examining the presence of attentional and interpretive biases in insomnia. However, they largely rely on abstracted reaction time tasks which remain distal from the experience of insomnia. Considering quantitative methodologies of this nature fail to capture the intimate nature and context of the 
individuals' experience of the disorder, a more comprehensive understanding may be gained through qualitative inquiry. Indeed, whilst qualitative research in insomnia has steadily increased over the years according to a recent review [20], the role of attentional and interpretive biases is yet to be examined from this perspective. As such, this study sought to examine, using a qualitative methodology, the role of attentional biases in insomnia. Specifically, this study will use semi-structured interviews and thematic analysis to gather introspective, in-depth data regarding the perception and nature of such biases amongst individuals meeting the diagnostic criteria for insomnia.

\section{Methods}

\subsection{Ethics}

Ethical approval was granted by the Sheffield Hallam University Research Ethics committee. All participants provided written informed consent prior to participation.

\subsection{Participants}

Participants were recruited from the general population through social media, 'call for participants' (website), and students from Sheffield Hallam university through the institutions course participation scheme. To be included in the study, all participants had to meet the DSM-5 diagnostic criteria for insomnia disorder [21]. Specifically, individuals with insomnia reported dissatisfaction with sleep characterised by either a difficulty initiating or maintaining sleep or early morning awakenings. Insomnia had to be present for three or more nights per week, for at least 3 months, and cause significant daytime impairment. Finally, these conditions had to be met despite adequate opportunity to sleep. A screening questionnaire determined eligibility and insomnia status. Individuals who reported symptoms of a sleep/ wake disorder other than insomnia, an existing psychiatric illness, a central nervous system disorder, use of medication that may affect sleep, prior head injury or current shift work were ineligible to participate. Of note, the SLEEP-50 [22] questionnaire was used to ensure the absence of a sleep/ wake disorder other than insomnia. The final sample consisted of 11 individuals with insomnia (nine female, mean age $=27.97$ years, $\mathrm{SD}=9.52$ years)

\subsection{Procedure}

Semi-structured interviews followed a topic guide which was developed by the research team. The interview questions were adapted from the Sleep-Associated Monitoring Index [23], Dysfunctional Beliefs and Attitudes about Sleep
Scale [24], and the Sleep-Related Behaviour Questionnaire [25]. Questions largely focused on four domains: (1) selective attention for sleep-related cues immediately upon waking; (2) selective attention for sleep-related cues through the day; (3) compensatory safety behaviours due to insomnia; (4) beliefs regarding the impact of insomnia, encompassing perceived effects on daily functioning, social interaction and physical appearance.

Interviews were conducted face to face by the two researchers (ZF, SK) in a quiet psychological laboratory testing room at Sheffield Hallam University. All participants were provided with the opportunity to ask questions before providing written informed consent. Interviews were audio recorded and then transcribed verbatim by the research team with any identifying information being removed at this stage.

\subsection{Analysis}

The data were analysed using an inductive and semantic thematic analysis. Here, we used the recursive process outlined by Braun and Clarke [26] to guide the analysis procedure. First, by repeatedly reading the transcripts, familiarity with the data was achieved. Following subsequent readings of individual transcripts, relevant words or phrases were highlighted to aid in developing codes. Transcripts were then manually coded through the identification of any features deemed interesting or relevant after several further reviews of the transcripts were made [27]. Next, initial themes were generated by grouping the identified codes based on their similarities and differences. Themes were compared across each transcript to explore any recurrent themes, inconsistencies and contradicting cases. Themes were refined throughout the analytic procedure via discussions with another researcher in the area of insomnia. Consequently, latent themes were named, and a thematic map was finalised.

\section{Results}

\subsection{Thematic Analysis}

The analysis procedure revealed two prominent, yet interrelated, themes: impairment to daytime functioning; and selective attention and interpretation. Within each main theme, several more specific sub-themes were identified (see Table 1). Direct quotes are provided to support themes.

\subsection{Impairment to Daytime Functioning}

\subsubsection{Burden of Mundane Tasks Amplified}

Almost all participants stressed the importance of sleep for optimal daytime functioning and frequently reported the 
Table 1 Determined themes and sub-themes yielded from Sect. "Thematic analysis"

\begin{tabular}{ll}
\hline Theme & Sub-themes \\
\hline $\begin{array}{l}\text { Impairment to daytime } \\
\text { functioning }\end{array}$ & Burden of mundane tasks amplified \\
$\begin{array}{l}\text { Selective attention and } \\
\text { monitoring }\end{array}$ & $\begin{array}{l}\text { Adaptation through compensatory behaviour } \\
\text { Self-assessment of sleep on awakening }\end{array}$ \\
& $\begin{array}{l}\text { Throughout the day } \\
\text { The appearance of insomnia }\end{array}$ \\
\hline
\end{tabular}

debilitating nature of insomnia on their waking lives. It was apparent that perceived difficulty initiating and maintaining asleep during the biological night was accompanied by increased efforts during the day to complete mundane tasks. Central to this was reported difficulty in getting out of the bed in the morning which was influenced by individual's perceptions of sleep quality the preceding night and the importance of the event in which they were required to wake up for (i.e., work, university study).

In my first year (at university), if I couldn't get to sleep, I just wouldn't go to university. [Pause] But this year (final year), I'm like no! - I need to go, and I think that is kind of like an incentive to get out of bed.

I've had times where I have cancelled plans because I have been too tired to go because I have only had a few hours' sleep the night before.

Following initial difficulty waking up, once an effort to start the day has been made, participants often went on to experience deficits in concentration through the day. This again was attributed to the poor nature of their sleep. Participants explained that their inability to concentrate acted as a barrier to optimal performance within the workplace, with one participant confessing to making mistakes at work as a result of their insomnia. Moreover, during study, some participants reported zoning out or falling asleep during lectures citing basic cognitive demands (e.g., reading, writing) as exhausting.

I work with people. When they talk to me I have to sit and nod and concentrate on what they're saying because I can feel myself [Pause] floating off and not paying attention because concentration is difficult.

Impaired social interaction was also highlighted, often as a consequence of their recurrent mood, being described as 'grumpy' or 'miserable'. As a result, participants frequently reported either avoiding or feeling withdrawn in most social situations, particularly after a night of poor sleep.

Everything takes so much effort. I think socialising is probably the harder part, so it does kind of isolate me a little because [Pause] dealing with allot of people in a room is draining anyway. But, then when you haven't exactly slept anyway it kind of amplifies it.
I can't be bothered to use flowery language. I just give facts. Sometimes people interpret that as being rude or abrupt and I'm not, I just have no energy.

\subsubsection{Adaptation Through Compensatory and Coping Behaviour}

Whilst participants described difficulty completing day-today activities, a number of strategies were reported which aimed to maximise daily functioning. These included the excessive consumption of excess, daytime napping, and planning ahead to optimise performance. One participant even reported choosing to live near their place of study to optimise attendance. Whilst another reported starting coursework months ahead of others to take capitalise on bouts of normality where they could indeed concentrate.

I'm probably a bit more robotic and a bit more structured. When I'm talking or responding [Pause]. I have strategies in place to break things down into smaller tasks if I'm not coping very well during the day.

All participants alluded to their reliance on caffeine when tackling the fatigue associated with insomnia, with one individual referring to the substance as their 'best friend'. Whilst it was reported that functionality would be minimal without its use, all participants demonstrated an awareness of potential dependence. In this case, those who perceived immunity to the effects reported ingesting more caffeine to obtain the desired effect. However, participants seemed unaware of the paradoxical effect which this consumption would have on metabolic activity and subsequent delay of sleep onset.

Similar to caffeine, some participants specified a tendency to sleep during the day to again tackle fatigue associated with the disorder. That said, some reported difficulty in initiating sleep during the biological day, consequently likening a successful nap to 'heaven'. In contrast, several participants displayed an awareness of the potential negative night-time effects after napping during the day. With that in mind, some reported restricting their nap to an hour with the belief that the experience of fatigue may be amplified with anything longer in duration.

I try not to take them (naps) because they are not meant to be very helpful. Because, obviously, I won't be able to sleep at night. Um, so I do try, I mean I really, really try and not have a nap at all but sometimes I just'.

\subsection{Selective Attention and Monitoring}

\subsubsection{Self-Assessment of Sleep on Awakening}

The majority of participants reported checking the clock to calculate how many hours of sleep were obtained on awakening. In addition, participants commonly assessed internal feelings and reported experiencing physical sensations 
related to tiredness, which were attributed to the poor nature of their sleep. Here, tiredness was conceptualised as feeling 'drained' and 'groggy', and was characterised by sore and heavy eyes, aches and pains in the body, and an inherent lack of energy. Subsequently, these assessments were used to draw conclusions regarding the length and quality of sleep obtained the previous night, as participants' extreme feelings of tiredness were interpreted as confirmation of a particularly poor night's sleep.

I kind of wake up and the first thing I pay attention too is how groggy I feel, because [Pause] when I am groggy, it means I haven't had quality of sleep.

When I wake up [Pause] my eyes are really heavy like sometimes my eyes are like quite stingy.

I focus on my internal cues, so I feel sick when I wake up, I have the shakes, my body aches, and my eyes sting.

This pattern of behaviour appears to extend to external physical cues on awakening. Participants reported examining their facial appearance in the mirror after getting out of bed, which was subsequently perceived in a negative manner with one participant noting that they 'look like a zombie'. Whilst some participants focused on the appearance of their skin and overall complexion, the region in and around the eyes appeared to be the focus for most.

If you saw me without make up on my eyes are so dark. Like, a girl of [Masked] years old should not have dark circles like I do. Like, they're really dipped in and my eyes are so tired. Sometimes I wake up and [Pause] they can have like a yellowy, red-y tone'.

When I look in the mirror, the first thing I'm drawn too are my eyes. They're like a big indicator of how tired I am. They make me look like I have three hours sleep, which wouldn't be wrong.

When I wake up in the morning, I dread to think what I look like. My eyes are always heavy when I wake up. Sometimes I look like I've had no sleep at all with the dark circles I have under my eyes.

\subsubsection{Throughout the Day}

Although participants commonly reported selective attention to sleep-related cues on awakening, the likelihood of continuing this through the day differed vastly. For some, the propensity to examine internal cues was augmented on days where physically or cognitively demanding tasks were required. Despite the apparent dissatisfaction with facial appearance on awakening, the likelihood of examining facial cues was largely dependent on convenience and when the opportunity presented itself. Moreover, some participants solely attended to internal cues throughout the day.

If I go to the bathroom, I will look at myself in the mirror and examine what my face looks like [Pause] more so look at my eyes.
I can't help but check up on how I look throughout the day. It's like if you know you look bad, you kind of want to monitor that, with hopes you don't start looking worse if that makes sense?.

That said, not all participants reported attending to such cues throughout the day. With one participant noting that they actively avoid such behaviour.

I try and avoid looking at myself throughout the day. If I look, it's just a reminder of how tired I actually am and look.

\subsubsection{The Appearance of Insomnia}

Almost all participants explained how they could recognise the appearance of tiredness and tell when other people had also slept poorly. Here, participants described sleep-deprived individuals as having: 'sunken eyes'; 'dark circles' (under the eyes); 'pale skin'; and 'dullness in their face'. Interestingly, participants also highlighted aspects of body language which served to infer poor sleep the previous night. This included being 'slumped' or appearing 'lethargic' and could even be determined by an individual's voice.

Their eyes [Pause] not even just looks but just how they are. Like, their body language. Like if they are sat slouched like I am now [Pause]. I haven't slept properly last night, and I'm really slouched. Even someone's voice sometimes'.

A number of participants admitted to drawing contrasts between the facial appearance of themselves and other people. Specifically, the eye area of other individuals appears to be examined for skin discolouration. That said, participants perceived their own facial appearance as appearing more tired. Indeed, one participant claimed that in comparison with other individuals, their facial appearance was ' $a w f u l$ ' and 'tired'.

I do find myself comparing myself to others, only on a physical basis. I think if you look at me and then look at someone else, it's clear who has had the better night's sleep.

I can tell I'm more tired than other people.

Furthermore, participants' beliefs surrounding their own facial appearance seemed to be reinforced by other individuals making comments about their facially tired appearance.

Yesterday I didn't put any make up on whatsoever, and [Pause] my friend was like 'are you alright?'.

I do get told that I look like a zombie.

\section{Discussion}

This study was the first to use a qualitative design to explore the role of daytime attentional biases in insomnia. Thematic analysis produced two prominent, yet interconnected, themes: impairment to daytime functioning, and selective attention and monitoring. 
In line with previous research, sleep was perceived to be the greatest influence for the ability to focus optimally during the day $[3,28]$. Considering the persistent reporting of poor sleep amongst this population, it is perhaps not surprising that all participants noted the experience of insomnia as being debilitating on their waking lives. Indeed, the apparent difficulty initiating and maintaining asleep during the biological night often lead participants to express increased effort during the day when complete mundane tasks and engaging in social interaction. This over-concern with sleep and perceived difficulty in competing daytime tasks and engaging in social interaction serves to influence counterproductive behaviour amongst participants. Specifically, as noted in cognitive models of insomnia (e.g., [4-6]), participants reported engaging in a number of counterproductive safety behaviours, e.g., increased caffeine intake, avoiding social situations to rest, increased time in bed through napping or attempting to sleep earlier and increased efforts to sleep to compensate for their sleep deficit. Paradoxically, engaging in these behaviours serves only to perpetuate and maintain the sleep disturbance.

The present outcomes also demonstrate that people with insomnia report consciously selectively attending to sleeprelated cues on awakening, and for some this behaviour extends throughout the day. Whilst this notion is supported by previous research [23, 29], the present data provide patient-level insight concerning the function of such behaviour. On awaking, the primary function of selective attention for internal (i.e., body sensations) and external (facial appearance, the clock) was to evaluate the quality and length of sleep obtained. Participants would generally report feeling tired and unrefreshed on awakening, perceiving aspects of their body as feeling sore and heavy. The face and particular the eyes and surrounding region appeared to be of particular external focus of attention for many. Here, facial dissatisfaction was highly prevalent, with eyes being described as being sunken, heavy, red or yellow in colour, and accompanied with large bags underneath.

In contrast, selective attention though the day was largely focused on internal physical sensations related to tiredness, for some this was increased on days where physically or cognitively demanding tasks were required. Whilst attention for facial features was prominent on awakening, whether this extended through the day depended on convenience and when the opportunity presented itself (e.g., using the mirror in bathroom breaks). Interestingly, the current outcomes show that individuals with insomnia are sensitive to the appearance of insomnia, not just in terms of self-perception, but when presented by other people. Participants correctly described a number of validated cues which serve to communicate the presence of sleep deprivation (i.e., heavy eyes, bags under eyes: [30, 31]). In addition, a number of cues yet to be research were also discussed (e.g., posture, voice). That said, participants still perceived their own facial appearance as appearing more tired when compared to other people. With that in mind, it may be postulated that individuals with insomnia attend to other people's facial tiredness cues to evaluate these features and subsequently draw comparisons to their own. Indeed, the face is commonly used in social perception by individuals to interpret another's internal state [32]. Therefore, those with insomnia may not only monitor and consequently perceive their own appearance in terms of an increased sleep deficit but may also observe others to use this information to inform their judgments in a negative way (e.g., I look exhausted compared to everyone else: [33]).

The current outcomes also provide insight into the previously determined relationship between insomnia and cut dissatisfaction with cutaneous body image and facial appearance [29, 34], [35] by providing a patient level account of this potentially mediating factor. As mentioned in the introduction, insomnia is partly maintained by selective attention and monitoring for cues that indicate the presence of a poor night's sleep [4-6]. Questionnaire studies evidence that individuals with insomnia report monitoring bodily sensations on waking and throughout the day for signs of impairment, which include tiredness (i.e., sore head, heavy eyes) resulting from a poor night's sleep. Here, the current results expand on these self-reports and experimental eye-tracking and reaction time research by producing patient level confirmation of the propensity to monitor one's own face, with a specific focus around the eye-region, for cues associated with tiredness (i.e., hanging eyelids, dark circles). This emphasis upon awakening and, possibly during the day, to confirm the nature of insomnia may contribute to increasing worry, arousal and distress as described in cognitive models of insomnia [4-6].

Individuals with insomnia commonly interpret negative characteristics of their physical self (i.e., facial cues of tiredness, facial complexion cutaneous features) in a negative way [34-36]. Considering this, normalising the perception of these characteristics may act as a novel treatment method. Indeed, feedback relating to a self-misperception of facial tiredness in insomnia has previously functioned to improve self-perception such that participants later interpreted themselves as appearing less tired [36]. An initiation of a more accurate self-perception amongst this population could work to eliminate a maintaining factor of insomnia (i.e., the propensity to negatively interpret physical cues consistent with a poor night's sleep). However, attention precedes interpretation [37]. To that end, recent research has verified the effectiveness of delivering attentional bias modification (ABM) immediately prior to bed as a treatment for poor sleeping individuals [38]. Here, poor sleepers provided with ABM displayed improved subjective sleep quality, reduced presleep arousal, and reduced sleep onset latency relative to poor-sleeping controls. Considering the reports and location 
of selective attention made amongst individuals with insomnia in the current study, future research should examine the efficacy of attentional bias modification using sleep-related facial images of participants in an effort to reduce selective attention to tiredness cues in this population.

Several limitations of the current study should be noted. First, the current outcomes can only be attributed to the small sample of eleven participants who completed the study and cannot be extrapolated to all individuals with insomnia. Second, the current sample consisted predominately of female participants with insomnia. As such, the current outcomes may not be fully generalisable to males. That said, it is relevant to note that women are more likely to experience insomnia when compared with men [39]. Despite this, this study was the first to examine daytime attentional biases in insomnia using qualitative analysis. The two themes identified, impairment to daytime functioning and selective attention, provide novel support for cognitive models for insomnia [4-6].

Funding This research was supported by the Department of Psychology, Sociology and Politics at Sheffield Hallam University.

\section{Compliance with ethical standards}

Conflict of interest No conflicts of interest declared in relation to this paper.

Open Access This article is distributed under the terms of the Creative Commons Attribution 4.0 International License (http://creativeco mmons.org/licenses/by/4.0/), which permits unrestricted use, distribution, and reproduction in any medium, provided you give appropriate credit to the original author(s) and the source, provide a link to the Creative Commons license, and indicate if changes were made.

\section{References}

1. Espie CA, Kyle SD, Hames P, Cyhlarova E, Benzeval M. The daytime impact of DSM-5 insomnia disorder: comparative analysis of insomnia subtypes from the Great British Sleep Survey. J Clin Psychiatry. 2012;73(12):e1478-84.

2. Morin CM, LeBlanc M, Daley M, Gregoire JP, Merette C. Epidemiology of insomnia: prevalence, self-help treatments, consultations, and determinants of help-seeking behaviors. Sleep Med. 2006;7(2):123-30.

3. Kyle SD, Espie CA, Morgan K. “... Not just a minor thing, it is something major, which stops you from functioning daily": quality of life and daytime functioning in insomnia. Behav Sleep Med. 2010;8(3):123-40.

4. Espie CA. Insomnia: conceptual issues in the development, persistence, and treatment of sleep disorder in adults. Annu Rev Psychol. 2002;53(1):215-43.

5. Espie CA, Broomfield NM, MacMahon KM, Macphee LM, Taylor LM. The attention-intention-effort pathway in the development of psychophysiologic insomnia: a theoretical review. Sleep Med Rev. 2006;10(4):215-45.
6. Harvey AG. A cognitive model of insomnia. Behav Res Ther. 2002;40(8):869-93.

7. Akram U, Beattie L, Ypsilanti A, Reidy J, Robson A, Chapman AJ, Barclay NL. Sleep-related attentional bias for tired faces in insomnia: evidence from a dot-probe paradigm. Behav Res Ther. 2018;30(103):18-23.

8. Akram U, Robson A, Ypsilanti A. Sleep-related attentional bias for faces depicting tiredness in insomnia: evidence from an eyetracking study. J Clin Sleep Med. 2018;14(06):959-65.

9. Barclay NL, Ellis JG. Sleep-related attentional bias in poor versus good sleepers is independent of affective valence. J Sleep Res. 2013;22(4):414-21.

10. Beattie L, Bindemann M, Kyle SD, Biello SM. Attention to beds in natural scenes by observers with insomnia symptoms. Behav Res Ther. 2017;1(92):51-6.

11. Jansson-Fröjmark M, Bermås M, Kjellén A. Attentional bias in insomnia: the dot-probe task with pictorial stimuli depicting daytime fatigue/malaise. Cognit Ther Res. 2013;37(3):534-46.

12. Lundh LG, Froding A, Gyllenhammar L, Broman JE, Hetta J. Cognitive bias and memory performance in patients with persistent insomnia. Cognit Behav Ther. 1997;26(1):27-35.

13. Macmahon KM, Broomfield NM, Espie CA. Attention bias for sleep-related stimuli in primary insomnia and delayed sleep phase syndrome using the dot-probe task. Sleep. 2006;29(11):1420-7.

14. Marchetti LM, Biello SM, Broomfield NM, Macmahon $\mathrm{KM}$, Espie CA. Who is pre-occupied with sleep? A comparison of attention bias in people with psychophysiological insomnia, delayed sleep phase syndrome and good sleepers using the induced change blindness paradigm. J Sleep Res. 2006;15(2):212-21.

15. Spiegelhalder K, Espie C, Nissen C, Riemann D. Sleep-related attentional bias in patients with primary insomnia compared with sleep experts and healthy controls. J Sleep Res. 2008;17(2):191-6.

16. Spiegelhalder K, Baglioni C, Regen W, Kyle SD, Nissen C, Hennig J, Doerr JP, Feige B, Riemann D. Brain reactivity and selective attention to sleep-related words in patients with chronic insomnia. Behav Sleep Med. 2016;15:1-5.

17. Woods H, Marchetti LM, Biello SM, Espie CA. The clock as a focus of selective attention in those with primary insomnia: an experimental study using a modified Posner paradigm. Behav Res Ther. 2009;47(3):231-6.

18. Woods HC, Scheepers C, Ross KA, Espie CA, Biello SM. What are you looking at? Moving toward an attentional timeline in insomnia: a novel semantic eye tracking study. Sleep. 2013;36(10):1491-9.

19. Harris K, Spiegelhalder K, Espie CA, MacMahon KM, Woods HC, Kyle SD. Sleep-related attentional bias in insomnia: a stateof-the-science review. Clin Psychol Rev. 2015;1(42):16-27.

20. Araújo T, Jarrin DC, Leanza Y, Vallières A, Morin CM. Qualitative studies of insomnia: current state of knowledge in the field. Sleep Med Rev. 2017;1(31):58-69.

21. American Psychiatric Association. Diagnostic and statistical manual of mental disorders (DSM- $\left.5^{\circledR}\right)$. American Psychiatric Pub; 201322

22. Spoormaker VI, Verbeek I, van den Bout J, Klip EC. Initial validation of the SLEEP-50 questionnaire. Behav Sleep Med. 2005;3(4):227-46.

23. Semler CN, Harvey AG. Monitoring for sleep-related threat: a pilot study of the Sleep Associated Monitoring Index (SAMI). Psychosom Med. 2004;66(2):242-50.

24. Morin CM, Vallières A, Ivers H. Dysfunctional beliefs and attitudes about sleep (DBAS): validation of a brief version (DBAS16). Sleep. 2007;30(11):1547-54. 
25. Ree MJ, Harvey AG. Investigating safety behaviours in insomnia: the development of the sleep-related behaviours questionnaire (SRBQ). Behav Change. 2004;21(1):26-36.

26. Braun V, Clarke V. Using thematic analysis in psychology. Qual Res Psychol. 2006;3(2):77-101.

27. Tuckett AG. Applying thematic analysis theory to practice: a researcher's experience. Contemp Nurse. 2005;19(1-2):75-87.

28. Buysse DJ, Thompson W, Scott J, Franzen PL, Germain A, Hall M, Moul DE, Nofzinger EA, Kupfer DJ. Daytime symptoms in primary insomnia: a prospective analysis using ecological momentary assessment. Sleep Med. 2007;8(3):198-208.

29. Akram U. Sleep associated monitoring on awakening mediates the relationship between cutaneous body image dissatisfaction and insomnia symptoms. Sleep Sci. 2017;10(2):92.

30. Knoll BI, Attkiss KJ, Persing JA. The influence of forehead, brow, and periorbital aesthetics on perceived expression in the youthful face. Plast Reconstr Surg. 2008;121(5):1793-802.

31. Sundelin T, Lekander M, Kecklund G, Van Someren EJ, Olsson A, Axelsson J. Cues of fatigue: effects of sleep deprivation on facial appearance. Sleep. 2013;36(9):1355-60.

32. Allison T, Puce A, McCarthy G. Social perception from visual cues: role of the STS region. Trends Cognit Sci. 2000;4(7):267-78.
33. Akram U, Ellis JG, Myachykov A, Barclay NL. Preferential attention towards the eye-region amongst individuals with insomnia. J Sleep Res. 2017;26(1):84-91.

34. Gupta MA, Gupta AK, Knapp K. Dissatisfaction with cutaneous body image is directly correlated with insomnia severity: a prospective study in a non-clinical sample. J Dermatol Treat. 2015;26(2):193-7.

35. Oyetakin-White P, Suggs A, Koo B, Matsui MS, Yarosh D, Cooper KD, Baron ED. Does poor sleep quality affect skin ageing? Clin Exp Dermatol. 2015;40(1):17-22.

36. Akram U, Ellis JG, Myachykov A, Barclay NL. Misperception of tiredness in young adults with insomnia. J Sleep Res. 2016;25(4):466-74.

37. Akram U. The face of tiredness in insomnia from the self-perspective: a focus on attentional and interpretative biases. J Sleep Res. 2018;27(3):e12657.

38. Milkins B, Notebaert L, MacLeod C, Clarke PJ. The potential benefits of targeted attentional bias modification on cognitive arousal and sleep quality in worry-related sleep disturbance. Clin Psychol Sci. 2016;4(6):1015-27.

39. Zhang B, Wing YK. Sex differences in insomnia: a meta-analysis. Sleep. 2006;29(1):85-93. 